\title{
Lo que escribimos, los que nos leen
}

2. Graciela Montaldo / Columbia University / gm2i68@columbia.edu

\section{Resumen}

Este artículo se propuso describir algunas prácticas de escritura y lectura en la escena contemporánea. Apunta a dos aspectos de la misma práctica: cierta experiencia histórica de la escritura crítica y las condiciones del trabajo crítico en el presente. Se detiene básicamente en los problemas y desafíos institucionales de la producción cultural contemporánea y en la forma que entendemos los cambios en los saberes. Lo hace en el contexto de los estudios latinoamericanos. Su hipótesis principal es que la férrea homogeneización institucional abre, sin embargo, espacios de fuga a la normalización escrituraria.

Palabras clave: campo cultural $\cdot$ literatura $\cdot$

contemporaneidad $\cdot$ crítica cultural $\cdot$ escritura y lectura

\section{Abstract}

This article is focused on the description of the practices writing and reading in the contemporary scene. It studies two levels of the same practice: an historical experience of the critical writing and the conditions of the critical work in the present moment. It basically interrogates the institutional circumstances and challenges of the contemporary cultural production and in the new ways to understand the new knowledges. It studies those issues in the context of Latin American studies. Its main argument is: the rigid institutional homogeneity opens up some leaks and uncertain spaces inside the system.

Key words: cultural field $\cdot$ literature $\cdot$ contemporary practices $\cdot$ critical thought $\cdot$ writing and reading
Toda investigación, deja hoy explícito su «lado B», la forma en que se construye y reflexiona sobre sí misma. No es algo que acontece hoy por primera vez, pero hoy se ha vuelto necesario escribir también sobre las circunstancias problemáticas que cruzan las varias capas institucionales que intervienen en la investigación. No se trata solo de qué hacemos sino de las condiciones que tenemos para hacerlo y de las formas en que llegamos a concretar nuestros trabajos. Pero cuando somos tan conscientes de lo que nos llevó a escribir esto o lo otro, de cómo construimos nuestros archivos y nuestros objetos, todo nos lleva a fijar la vista en quienes van
Fecha de recepción: 29/06/2016

Fecha de aceptación: 2/08/2016 
a leer lo que escribimos. En un punto, escribimos eso para ellos/as. Hay, siempre, primeros lectores y lectoras institucionales (evaluadores, editores, colegas), pero después, ¿qué pasa con aquello que escribimos? La pregunta no se dirige a cuestionar o identificar un «target» sino a explorar la práctica misma de la escritura y la lectura conjuntamente. Por ello, la pregunta por cómo leemos, en qué formatos, con qué expectativas, incluso, por qué leemos debe ser formulada. La pregunta por la escritura marcó los estudios críticos desde los años 6o. Desde antes, ciertamente, pero fue en los ańos 60 , con el estructuralismo en alza, que la escritura y todo lo que ella implicaba por entonces como centro de la experimentación crítica, se volvió eje de reflexión, discusión y, especialmente, auto-reflexión.

Creo que por eso hoy resulta interesante saber qué y cómo leemos. Me pregunto qué clase de objetos constituyen hoy nuestras bibliotecas, materiales y virtuales, reconvertidas en archivos. La mayoría de los críticos, entrenados en literatura, nos fuimos inclinando hacia otras opciones desde principios de los años 90. No fue sólo presión de la aparición de los estudios culturales en Inglaterra/ Estados Unidos. Esa presión que, sin duda existió, quizás vino a canalizar - e instrumentalizar en términos de las instituciones académicas - una insatisfacción muy presente ya en los años 90: la insatisfacción con las disciplinas tal como continuaban siendo formuladas. Leer la literatura sólo en relación con la literatura, por ejemplo, resultaba una actividad relativamente pobre cuando se pensaba a la literatura en un contexto algo mayor. La convicción de que los escritores no leían solo literatura y que escribían sus textos con experiencias (personales y, especialmente, culturales) que excedían las bibliotecas personales o generacionales, tenía que terminar por imponerse. Parece una conclusión obvia, pero no lo era tanto para una disciplina que a fuerza a análisis textuales, close readings, se había especializado en la interpretación de la forma escrita, en la consumación de la autonomía, en el placer de la escritura. Abolida (o debilitada) esa restricción, el mundo entero aparecía como disponible a la hora de pensar la literatura, el arte, la música y por eso se impuso con tanta fuerza la idea de "cultura», que vino a implantarse, como una suerte de "paraguas» contenedor, a las diferentes prácticas artísticas al mismo tiempo que al estudio que se hacía de ellas. A su vez, la estética, que había sido "descubierta» en muchos otros rubros de la praxis cultural, le quitaba al arte su exclusividad y habilitaba su lectura en nuevos contextos, en correlación con otras experiencias.

Fue entonces cuando las bibliotecas profesionales comenzaron a cambiar notoriamente. Digo bibliotecas en un sentido activo; antes también las bibliotecas incluían libros de arte, filosofía, música, medios; pero poco a poco los libros comenzaron a mezclarse y los libros mismos se volvieron más híbridos. Con esos cambios, las discusiones y las posibilidades de imaginar nuevos objetos también se desarrollaron en múltiples direcciones. El entrenamiento más generalizado en la escritura generó también cierta desacralización a la hora de ponerse a escribir. El proceso fue posible gracias a la aparición de muchas nuevas formas de publicaciones, desde las editoriales independientes, interesadas en trabajos novedosos, 
hasta las revistas académicas que cumplían con el doble papel de difundir el saber producido en las instituciones universitarias a la vez que garantizar —a través de la normalización del curriculum vitae - la formación de una clase de investigadores profesionales, con parámetros globales. Los subsidios para publicaciones vinieron a colaborar oportunamente con este sistema.

Esta serie de cambios afectó de manera similar a la producción literaria y a la crítica. Si pensamos en la literatura, hasta los años 90, las editoriales que publicaban literatura argentina eran muy pocas y tenían exigencias insondables para los escritores jóvenes, a los que les costaba mucho publicar su primera novela (e, incluso, seguir publicando). Con las sucesivas crisis, esas editoriales - que pertenecían, además, a conglomerados transnacionales - prácticamente dejaron de publicar literatura nueva y sólo se quedaron con los escritores canónicos o de éxito garantizado. Lo que terminó siendo positivo de aquella situación fue que pudieron proliferar editoriales independientes, que generaron espacios nuevos, no tan jerárquicos ni basados en criterios meramente mercantiles. Las políticas neoliberales, que en los años 90 destruyeron gran parte de las industrias nacionales en Argentina, desmantelaron el aparato editorial local, que fue vendido a empresas trasnacionales. La ausencia de grandes editoriales comerciales de capitales argentinos, que no respondieran a los catálogos de los conglomerados, generó un movimiento importante de editoriales «alternativas», de pequeño volumen, que ocuparon el lugar que dejaron vacío las editoriales comerciales: ellas editaron la literatura y los ensayos de escritores y críticos locales jóvenes, traducciones de ensayos o libros académicos de autores poco comerciales. Gran parte de la renovación estética, crítica, filosófica, se registró en un circuito cultural de sellos editoriales pequeños, no estandarizados, que se vendían solo en algunas librerías o en centros culturales y que progresivamente se fueron imponiendo.

Este desarrollo no se hizo al margen del mercado, sino en una suerte de otra forma de circulación mercantil, donde las escalas eran más reducidas y el público más focalizado: pocos ejemplares, distribución muy especializada en algunos circuitos alternativos, un catálogo algo más sofisticado y, en algunos casos, un cuidado especial por la materialidad del libro. La literatura entró en otro circuito; extrañada del gran público, se enfocó en el circuito más reducido de los lectores especializados. En medio del desarrollo de las nuevas tecnologías, del impacto de los blogs entre jóvenes escritores, críticos y lectores y la posibilidad de dar a conocer las obras casi inmediatamente a través de internet (posteriormente a través de los libros electrónicos), estas editoriales mantuvieron vivo un circuito en el que la literatura y la crítica decidieron trabajar desde un lugar de minoridad, oponiéndose (o, por lo menos, colocándose al margen) simultáneamente a las ediciones exclusivamente comerciales y a la circulación vía internet. La producción cultural sofisticada ya no aspiró a llegar a todos, a imponerse sino a interpelar a sus lectores, sus iguales. Las nuevas editoriales y los escritores y críticos que publicaron en ellas (muchos de los cuales participaban también de los circuitos convencionales) eligieron mantener una práctica que recuperaba experiencias muy modernas 
como, por ejemplo, impulsar una estética y un tipo de crítica particular (desarrollar un catálogo relativamente homogéneo), la importancia de lo artesanal (cuidar los diseńos de portadas y la materialidad del libro), los nombres y textos relativamente alternativos (que no respondían a las precisas demandas del mercado de ese momento, dirigido por los grupos editoriales mayores).

El limitado pero creciente movimiento editorial implicó no sólo a la literatura, la crítica y el ensayo; galerías de arte, salas de teatro y música, grupos de cine, centros de experimentación estética y cultural, generaron un circuito en donde fue posible cruzar prácticas y disciplinas, en donde los mismos actores funcionaban en más de un tipo de producción y, en muchos casos, las mezclaban. Las revistas digitales $y$, en otro sentido, las redes sociales, vinieron a agregar nuevos canales de circulación y, por tanto, de interlocución. Subsidios universitarios, premios, financiamientos institucionales y becas, permitieron también financiar revistas y libros. Si la escritura dejó de ser un problema central, si la publicación se volvió profesional, si las investigaciones comenzaron a circular entre una comunidad académica ampliada, como en un movimiento homeopático, la lectura comenzó a ocupar el lugar de aquello que necesitaba ser compensado, pensado ahora como el lugar posible del problema.

Creo que toda esta situación hizo que la escritura crítica — pero no sólo ellafluyera con cierta naturalidad. Es cierto que dejó de reclamar la complejidad que la había caracterizado en décadas anteriores, la sofisticación que la distinguía como una práctica escrituraria específica y no subsidiaria de la literatura. Pero se hizo más dialógica. La escritura crítica se hizo más legible, aunque no tuviera lectores (definidos). Creo que no se trata de una paradoja sino de las nuevas condiciones de legibilidad, cuando el acento está puesto en la circulación, entendida menos como recepción o lectura que como consumo. Creo que conviene no descuidar este aspecto. La crítica, que siempre fue una práctica minoritaria respecto de la literatura, comenzó también a formar parte del circuito del consumo. Paradójicamente, fueron las editoriales pequeñas y menos comerciales las que reactivaron el consumo cultural minoritario, apoyado — como dijimos- por blogs y revistas electrónicas.

Al afianzarse las instituciones, al profesionalizarse las prácticas, ambas tienden a conservarse y reproducirse mejor. Se ha dicho que la crítica hoy autoabastece y reproduce un circuito académico restringido y solipsista. ¿Qué no tiene lectores? ¿Quién los tiene? Las tiradas de los libros de literatura (que no son best-sellers) son muy limitadas; los libros críticos y las revistas especializadas tienen una circulación aún menor. La aspiración a un público ampliado, que se amplía, no parece formar parte del horizonte de buena parte de las escrituras contemporáneas aunque sea prerrogativa del mercado editorial. Quienes escriben y publican en el escenario contemporáneo suelen saber que la cuestión no es tener muchos lectores sino los lectores adecuados. Si las instituciones reproducen (objetos de investigación, bibliografías, a sí mismas), las comunidades que leen diseminan y conectan sentidos y temas, ensamblan prácticas de lectura diferentes y las ponen en movi- 
miento. Muchas de ellas vuelven a las instituciones a través de programas de clase, bibliografías, pero transformadas por un valor ganado en circuitos de discusiones.

La tradicional imagen de la mesa de luz con pilas de libros acumulados, que no se tiene tiempo de leer, sigue asomando como justificación o excusa cada vez que hay que confesar que no leímos tal libro. Pero no sé cuán real es. Leemos o «miramos» ciertos libros (cada vez se publican más libros especializados). A veces, no necesitamos leerlos para saber lo que son. Mencioné antes que creía que la idea de archivo había reemplazado la de biblioteca. Como todo cambio terminológico, se trata en realidad, de un cambio en las prácticas de la experiencia, en este caso, de investigación y de lectura y, por lo tanto, en la forma en que escribimos. El archivo es el acopio, el uso, lo instituyente y conservador (para Jacques Derrida) y la máquina de sacralizar y aplebeyar los objetos y la experiencia (para Boris Groys). Al archivo no hay (necesariamente) que leerlo, sólo construirlo, pensarlo, analizarlo. Pero no pertenece al ámbito de la lectura en términos tradicionales. Nuestras investigaciones, cada vez más, invocan al archivo, lo conforman y, por tanto, se alejan de una relación directa con la escritura: no siempre la escritura es la mejor forma de comunicar los resultados de una investigación. Ya no sorprende cuando se habla de la actividad crítica como una actividad «curatorial», es decir, "hacer algo» con la literatura: una edición, una antología, una exhibición, un documental, un evento. Los críticos no estamos sólo atados a la escritura sino que podemos experimentar más allá de los límites de la revista, el libro, la clase.

¿A quiénes leemos? ¿Cómo podemos hacer para leer todo lo que tenemos disponible, en la «mesa de luz»? La opción de encontrar en internet información que no cesa (sea veraz, confirmada, no corroborada, falaz), que se reproduce, que parece autogenerarse, que centra su principal valor en la «disponibilidad», que permite combinar diferentes medios, ya ha arrasado con la capacidad de leer en el sentido más tradicional. Y le otorga al libro (pero, básicamente, a las investigaciones) otros valores. Lecturas transversales, por palabras clave, por buscadores, concentran el foco en lo informativo. En la red, los argumentos tienden a desaparecer en función de una acumulación de información, "data», que viene a llenar una función de satisfacción en la mera búsqueda. A esto habría que sumar las licencias de Creative Commons, copyleft y las plataformas digitales, que ofrecen nuevas posibilidades de diseminar ideas (de muchos modos, pero esencialmente por escrito pero con componentes visuales). A los materiales y archivos digitalizados, ahora se agrega la posibilidad de la circulación digital. Con esta disponibilidad de materiales, también la práctica de la escritura se agita, es más «fácil», más rápida, más instantánea. No se puede - y nadie quiere ni lo intenta- competir con lo que ofrece la red que, por lo demás, está fuera de cualquier pretensión crítica. Pero se pueden construir alternativas y servirse de todo aquello que nos ofrece la nueva circulación. No hay cómo no responder al impacto de lo nuevo.

La experiencia de participar en algunos de los sitios Academic Commons, que comenzaron a proliferar, puede dar algunas ideas. Cada mes, el servidor entrega un resumen de las visitas que recibieron tus textos, la cantidad de gente que bajó 
el pdf del artículo, quienes sólo lo leyeron; es un resumen que, como si se tratase de una cuenta bancaria, o de tarjeta de crédito, convierte, mes a mes, la lectura también en "data». Esa anonimia ha dejado de ser perturbadora; es, simplemente, una condición más de nuestro presente. $\mathrm{Y}$ ha dejado de perturbar porque, aunque no sepamos quiénes nos leen, sabemos cuántos lo han intentado. Aquí ha habido un cambio. Cuando no sabemos quiénes nos leen, es difícil decir para quién escribimos pero podemos estar seguros de que tenemos lectores/as. Aquí las comunidades lectoras no son fácilmente identificables porque los buscadores de internet tienen su propio sistema de asociaciones, que hacen emerger nombres y temas cuando menos se los espera, que trabajan con algoritmos que buscan según otras lógicas, que no pueden seguir los parámetros de una disciplina sino los de una combinatoria. También encuentran coincidencias más o menos esperables, obviamente, pero lo novedoso es, en todo caso, lo inesperado. En realidad, en internet, no buscamos lo que queremos encontrar sino que intentamos que lo menos tradicional nos salga al encuentro.

Es en este sentido que creo que la experiencia de lectura se ha diversificado tanto que afecta, necesariamente, la escritura. No leemos con la misma disciplina que antes de la red; no escribimos con las mismas pretensiones. Ya hace tiempo que vivimos en otro mundo. A la lectura tradicional se agrega hoy el modo más aleatorio de lo disponible en los innumerables sitios, el más frenético de los buscadores, el más distraído de la intermedialidad. No importa mucho qué pensemos de Wikipedia o de Google u otros buscadores; allí están siempre para empezar una búsqueda sobre lo desconocido, para corroborar un dato, para resolver una duda, para limitar un problema. Ya casi nadie se excusa de usarla.

Para mi sorpresa, después de este excurso, me doy cuenta de que quizás sí pueda responder la pregunta inicial, quizás no es tan oscura como me pareció al comienzo. Creo que escribimos, como siempre, para los que nos leen, aunque no sepamos quienes son ni serán. Escribimos, también, porque —aunque de maneras muy diferentes - seguimos leyendo. Leemos porque la escritura ha seguido buscando caminos nuevos, ha respondido creativamente a los desafíos y a la amenaza de su sustitución; y la crítica, como práctica, se ha ido transformando. Hemos naturalizado la escritura, me parece, como una práctica más de nuestra lectura y en su intersección nos reconocemos.

\section{Instituciones}

Si esto es así, de todos modos no deberíamos perder de vista que casi la totalidad de nuestra práctica está mediada por las instituciones culturales; ellas son las que, en los hechos, permiten que todo funcione en los términos previstos para reproducir un determinado sistema educativo, cultural, estético. Su función es la reproducción. Y cualquier práctica crítica se mueve también dentro de ellas. Las instituciones no siempre tienen la misma relación con el Estado ni con el mercado; se instalan en un arco muy variado que abarca desde aquellas prácticas que explícitamente responden a ellos (premios nacionales, premios de las editoriales, 
canon escolar, producción de best-sellers, programas universitarios) a aquellas otras que oponen alguna resistencia o se conciben completamente en contra de ambos. Las instituciones - ya lo había señalado muy claramente Michel Foucault - son los instrumentos de la capilaridad que transitan las formas del poder (o del contrapoder), las formas modernas en que se transmiten las órdenes sociales, vengan del Estado, de grupos corporativos, de grupos de resistencia política o de grupos más laxos, pero que quieren tener alguna forma de intervención social. Son, entonces, espacios donde se articula la acción y, en la cultura contemporánea, después de adquirir un gran protagonismo (la redefinición de los museos, los premios editoriales, las bienales de arte, los programas de estudio de literatura e, incluso, los nuevos programas de «escritura creativa», por ejemplo), están mostrando una cara más versátil. Dentro de las instituciones donde la literatura se desarrolla, hay dos escenarios en los que me interesa poner énfasis: las editoriales alternativas y las universidades. Creo que habría que pensar en ambas como espacios de producción, no meramente como mediaciones entre autor, obra y lector sino como espacios activos donde la escritura también se acciona, se pone en movimiento y se produce.

El discurso crítico, especialmente el que se desarrolla en las universidades ha apoyado estos procesos de consolidación de un circuito que comenzó siendo alternativo. Si bien el canon no ha perdido su lugar hegemónico en la Academia, desde el ingreso de los estudios culturales a fines de los 8o, las formas alternativas al canon fueron ganando espacios muy rápidamente, canonizándose en algunos casos, generando zonas de novedad en otros. Por lo demás, las universidades se han vuelto globales, al ritmo de creación de un mercado académico. Congresos internacionales, publicaciones con los mismos o parecidos criterios de evaluación, acceso a e-books (o distribución informal de textos en formatos como pdf) generan también una dinámica que pone en contacto el discurso crítico con toda la novedad del circuito alternativo. Con vocación alternativa ella misma, la academia, que hace tiempo suscribió el desarrollo de los estudios culturales como forma de abandonar la filología y establecer cruces transdisciplinarios, se acopla al movimiento de «descanonizar» la crítica. Al hacerlo, contribuye a formar parte de la red que otorga prestigio a las nuevas producciones toda vez que se propone identificar nuevos objetos de estudio. Como institución, la academia solía ir muy por detrás de los movimientos del mercado y de la propia institución literaria; pero ya se ha ido acoplando a los ritmos más rápidos de lo contemporáneo. El caso argentino es indicativo pues una buena parte de los escritores jóvenes proviene de la carrera de Letras. A tal punto se produce la interrelación que en 2016 se creó, con enorme éxito de matriculación, la carrera «Licenciatura en artes de la escritura» en la Universidad Nacional de las Artes. Además, las carreras de Letras siguen estando entre la preferencia de gran cantidad de estudiantes.

Las universidades normalizaron un tipo de trabajo crítico que conjugó escritura, ensayo, teoría. Esta confluencia, esta dispersión y circulación de contenidos críticos y teóricos entre poblaciones amplias de estudiantes en universidades masivas, creó la paradoja de ver el desarrollo de un saber y un discurso que se 
expandieron más allá de su uso profesional. Esta paradoja constituyó un campo que, en la academia, conforma el estado actual de la crítica de la literatura (y muy probablemente a la literatura misma): la forma en que hoy se puede ser alternativos dentro de las instituciones que, por no representar un espacio unificado de saber, pueden interactuar de manera más libre con otras formas de producción de saber. Pero, al mismo tiempo, ponen en evidencia hasta qué punto la «cultura» (y también la política) ha desarrollado sus campos alternativos en el interior mismo de institucionalidad.

Si bien no se puede generalizar sobre los tópicos que se estudian en la academia, sin embargo, podemos decir que está sujeta a ciertas demandas disciplinarias que ordenan, clasifican y promueven temas. Basta mirar los programas de congresos como los de la Latin American Studies Association (LASA). Esta institución profesional de Estados Unidos, como hay tantas en el país según las disciplinas, fue fundada en 1966. En los últimos años sufrió un gran incremento en el número de miembros y también en la cantidad de propuestas para congreso (a raíz de lo cual, en el año 2012 se cambió la frecuencia de las reuniones: de I8 meses a I2 para dar cabida a todos los que querían participar). Aunque en las primeras décadas la membresía no estaba limitada a académicos de USA, el número de latinoamericanos residentes en América Latina era muy bajo pero, en los últimos ańos, ha crecido de manera sorprendente. En menor medida también creció la membresía de latinoamericanistas europeos y asiáticos. En su página web aparece la siguiente declaración:

La Asociación de Estudios Latinoamericanos (LASA) es la asociación profesional más grande del mundo que reúne a individuos e instituciones dedicados al estudio de Latinoamérica. Con más de 7000 miembros, de los cuales el cuarenta y cinco por ciento reside fuera de Estados Unidos, LASA es la asociación que reúne a los expertos en Latinoamérica de todas las disciplinas y a diversas áreas ocupacionales en todo el mundo.

Ese cuarenta y cinco por ciento, que crece, ocupa lugares en el esquema de gobierno de la institución y los congresos que, en los últimos años, se hacen tanto en Estados Unidos como América Latina. Las disciplinas que tienen mayor representación son ciencias políticas, en primer lugar y literatura en segundo (le siguen historia, antropología y arqueología y sociología). En estos congresos es posible encontrarse cada año con una actualización de los temas de investigación predominantes. Ellos funcionan como la superficie de contacto de los trabajos que se realizan especialmente en Estados Unidos y los países de América Latina y, obviamente, tienden a homogeneizar los intereses y propuestas. Cualquier asociado puede proponer una mesa; pero las propuestas están sujetas a la aprobación de un comité de expertos que quienes presiden el congreso nombran cada ańo. La selección se hace teniendo en cuenta los criterios profesionales que regulan la producción de un saber que cada vez es más parecido en todos los centros universitarios. Nuevas experiencias, como LASA-Sur, sirven para dislocar el eje 
geopolítico de la Asociación y generar espacios alternativos aunque es probable que deban entrar a la regulación general de la institución para garantizar su continuidad. Los congresos son una parte importante, pero lo son aún más las revistas especializadas. También ellas se ven obligadas, por cuestiones profesionales, a uniformar criterios de calidad académica y, con ello, temas de investigación.

Casi nadie, en el mundo académico, quiere quedar fuera de los parámetros que garantizan permanencia en el trabajo y continuidad en la investigación. Las revistas online, que han ganado prestigio con los años, son una forma de difusión que también ha cambiado el carácter nacional de las publicaciones y las ha abierto a un diálogo profesional-transnacional. Becas, acceso a bibliotecas online, proyectos en colaboración, pasantías, completan el cuadro de constitución de una suerte de lengua franca de la crítica literaria en español que, a la vez que crea debates transnacionales y transdisciplinarios, genera una notable homogeneización temática. Como siempre, existen las posibilidades de evadir las nuevas hegemonías a través de nuevos objetos pero no todos los actores del campo crítico pueden tomar esos riesgos por las precarias condiciones de trabajo en que muchos se encuentran. El mercado académico mueve, sin embargo, mucha gente. Y con ella, los temas viajan y se reproducen.

$\mathrm{Si}$ atendemos a esta suerte de estado de la cuestión, vemos que se ha ido estableciendo cierta idea de homogeneidad pero que, al mismo tiempo, las condiciones de centralización del mercado (editorial y académico) han generado también nuevas posibilidades de crear alternativas a la uniformidad del paisaje literario. Creo que esas alternativas, extremadamente variadas como pueden ser, tienen algunos aspectos en común. Me interesa mencionar, para concluir, dos: la idea de comunidad y el carácter proyectual de muchos emprendimientos. Construir comunidad podría ser el signo de una alternativa a las presiones de uniformidad; el carácter proyectual dinamiza las nuevas prácticas de modo que puedan siquiera intentar evadir la casi obligatoria institucionalización. Hubo un tiempo en que la pretensión de todo grupo estético o intelectual era imponerse, ganar un territorio y establecerse. Hoy, cuando casi todo es pasible de ser colonizado por el mercado o las instituciones, trabajar con la conciencia de la deserción, de ser capaz de abandonar lo que se ha creado, es un desafío que muchos intentan asumir (la idea de deserción la desarrolla Paolo Virno como una forma de crear alternativas a las políticas de integración o de confrontación). Y en ese desafío puede residir la posibilidad de crear alternativas. Sí se puede hacer, en la literatura y en la academia, como lo demuestran muchas prácticas contemporáneas.

Politizar tiempo y espacio simultáneamente puede ser un ejercicio de desclasificación de una historia que se constituye a través de múltiples unidades de lucha. Si alguno hay, ese podría ser el no-lugar de las humanidades hoy, un ejercicio de huida de las disciplinas. La paradoja de nuestro tiempo, lo que no deja de asombrar, es cómo las prácticas de la institucionalización y de resistencia conviven en nuestras escrituras y lecturas, conviven en la reconstitución de saberes y en el reacomodamiento de la vida cultural contemporánea. 


\section{Bibliografía}

Derrida, Jacques (1996). Mal de Archivo. Una impresión freudiana. Valencia: Trotta.

Foucault, Michel (1984). La arqueología del saber. Buenos Aires: Siglo XXI.

Groys, Boris. «Under Suspicion: a Phenomenology of the Media». Archive Public [en línea]. Consultado

el 20 de mayo de 2016 en https://archive- public.wordpress.com/texts/boris-groys/

LASA https://lasa.international.pitt.edu/eng/

VIrno, PaOlo (2002). Esercizi di Esodo. Linguaggio e azione politica. Verona: ombre corte. 